\title{
Effect of Selenium on Weight Change of HIV 1 Positive Pre-puberty Treatment Naive Children: Tick Phenomenon
}

\author{
Otieno Samwel Boaz ${ }^{1}$, Were Fred ${ }^{2}$, Kabiru Ephantus Wanjoi ${ }^{3}$ \\ ${ }^{1}$ Community Health Department, Faucalty of health Sciences, Greatlakes University, Nairobi, Kenya \\ ${ }^{2}$ Department of Pediatrics, School of Medicine, University of Nairobi, Nairobi, Kenya \\ ${ }^{3}$ School of Public Health, Kenyatta University, Nairobi, Kenya
}

Email address:

samwelbotieno@yahoo.com (O. S. Boaz)

\section{To cite this article:}

Otieno Samwel Boaz, Were Fred, Kabiru Ephantus Wanjoi. Effect of Selenium on Weight Change of HIV 1 Positive Pre-puberty Treatment Naive Children: Tick Phenomenon. Journal of Health and Environmental Research. Vol. 7, No. 3, 2021, pp. 134-138.

doi: $10.11648 /$ j.jher.20210703.13

Received: July 1, 2021; Accepted: July 10, 2021; Published: July 23, 2021

\begin{abstract}
Background: Seleno-proteins, Iodothyronine 5' dehydronases (DIO) affect the function of thyroid gland. The expression of receptors of DIOs are affected by sex hormones (Oestradiol and Testosterone). The objective of this research therefore was to study differential effect of these hormones in boys and girls (Tick-Phenomenon). Methods: Fifty HIV 1 positive, treatment Naïve children, were enrolled for the study. Half 25 were given fixed dose of $50 \mu$ gm yeast selenium while the matched 25 were on control. Weight was taken at 0,3 and 6 months. Results: Children on selenium had weight gain of $2.5 \mathrm{Kg}$ at six months. The weight for age $\mathrm{Z}$ score increased above $-2 \mathrm{SDs}$ cut off point at six months among children on selenium, in all age categories \{, 3-5 years $1.20 \pm 2.45,6-8$ years $0.19 \pm 0.880,9-15$ years $0.97 \pm 1.22\}$. In the matched Controls there was a decrease in WAZ in all the age categories to below -2 SDs at six months \{, 3-5 years $-2.218 \pm 1.46,6-8$ years $2.95 . \pm 3.10,9-15-2.30 \pm 1.240\}$. There was a significant WAZ difference between controls and selenium group at six months $\{\mathrm{F}$ $(5,12)=5.758, \mathrm{P}=0.006\}$. Female children on selenium initially had a decrease and then sharp increase in WAZ (Tick Phenomenon), compared to the males who had a gradual increase in WAZ. Conclusion: It can be concluded that intake of yeast Selenium led to significant increase in weight for age $\mathrm{Z}$ score at six months and that there is gender related differences in weight change between HIV 1 positive female and male children on test and controls, the females showing Tick phenomenon.
\end{abstract}

Keywords: Selenium, Children, Hormones, Pre-puberty, Weight Change, Children

\section{Introduction}

Selenium is ingested in various forms $[2,6]$ These forms vary from natural forms in diet to inorganic forms and biofortified foods. Intake from natural forms varies with diet [2] [4]. When taken selenium is easily absorbed into the blood in upper parts of intestine [17]. The amino acid selenium, selenomethionine, methylselenocystein, and selenocystein are absorbed through endothelial cells membrane by amino acid transporters $\mathrm{B}^{\circ}$ and borBAT system, while the selenate is transported by SLC26 system [2]. All forms of selenium are converted to selenide $\mathrm{H}_{2} \mathrm{Se}$ in endothelial cells [3]. Selemethionine and other species are converted to Selenocystine species, which is further converted to selenide, by action of selenocystein $\beta$-lyse [2]. Selenite are converted to selenide through an action of theorodoxine reductase.

Liver is the central organ in selenium metabolism [3] and the selenoproteins are in equilibrium with selenide pool [2] their degradation leads to selenide production and maintenance of the level (hepatic pool). Selenide is converted to selenoprotein $\mathrm{P}$ through action of selenophosphate synthetase [14]. The excretion of selenium is by conversion into selenide sugar and through Urine (selenuria) or by conversion into dimethyl selenide and excretion through breath and feces and trimethylselenium through urine (selenuria) [6].

Selenium is transported as SePP1 [6], which is a protein of 366 amino acids, [2], synthesized by hepatocytes and excreted in plasma [3]. SePP exists in two isoforms $60 \mathrm{kDa}$ and a $50 \mathrm{kDa}$ [9]. It has two terminals, the $\mathrm{N}$ terminal has 244 amino acids folded with one selenocystine residue, while 
the C-terminal domain has 122 amino acids and has 9 SePP [2]. The $\mathrm{N}$ terminal is responsible for thiol action, while the $\mathrm{C}$ terminal is responsible for the transport of selenium [2]. $\mathrm{SePP}$ is responsible for selenium transport to various organs in the human body [6, 5] Besides transport $\mathrm{SePP}$ is responsible for chelating heavy metals in the body, forming non-toxic complexes. The $60 \mathrm{kDa}$ isoform delivers selenium to body tissues, while $50 \mathrm{kDa}$ is involved in redox and signaling [18]. In plasma compartment SePP constitute about $60 \%$ of all selenium, and is responsive to dietary selenium [26] while GSH-px constitute 9-17\%. The SePP gene exists in two polymorphs, this determines amount of selenium delivered to the body up on intake. The levels of selenium is higher in the body among CC homozygotes compared to TTallele [9]. The uptake of selenium from Se-PP in various organs is by a receptor mediated-endocytosis [3]. In the kidney the uptake is by megalin receptors on brush side of endothelial cells [25], while sertoli cells in testis the uptake is by apoliprotein-E Receptors-2 [3]. Thyroid gland is thought to have alternative supply of selenium independent of SePP [2]. The selenium is found in high concentration in vital organs which include the liver, the kidney cortex, and in blood but in very low concentration in brain, muscles, pancreas, and pituitary [19, 20]. Liver is the central organ in metabolism of selenium [18], it receives selenium through the portal vein and takes up other forms from systemic circulation [3].

\section{Selenium and Weight Change}

Human genome codes for 25 seleno-proteins [18]. The thyroid gland is one of the body tissues with the highest levels of seleno-protein concentrations, [8]. This is due to presence of various species of seleno-proteins (GSH-px1, GSH-px2, GSHpx 4, Theorodoxine reductases 1 and 2 (TXNRD1, and TXNRD2, Diodonases 1 and 2 (DIO1 and DIO2) Sepp 15, SePP, and selenoprotein $M$ and $S$ ) in thyrocites. The combined deficiency of selenium, iodine and presence of thiocyanate leads to myxidematous cretinism [8]. In the thyroid hormones, selenocystein ( $\mathrm{SeCy}$ ) carries the active sites of the selenoproteins, in the two iso-forms of iodothyronine 5' deiodonases (DIOs).

Selenium is needed at all stages of early child development [7], the deficiency leads to myxidematous cretinism during late pregnancy and early childhood, or neurological cretinism in early pregnancy [7].

The weight change is associated with protein synthesis and deposition in the body. Selenium is part of a group of selenoenzymes Iodothyronine 5' deiodonases, produced in liver and thyroid gland. The DIO 1 is largely expressed in hepatocytes in endoplasmic reticulum membrane facing cytosol and in kidney and thyroid gland basal membrane [7]. TSH and cAMP increase its activity [7]. Corticosteroids enhances expression of DIO, in liver and pituitary gland $[11,7]$ Hunger and low selenium levels decreases expression of DIO. It catalyzes activation of $\mathrm{T}_{4}$ to the thyroid hormone 33 ' -5 Triiodothyronine [7], which is largely controlled at target cell level by regulation of intracellular DIO activities [23]. The hormone 3 3'-5 T3 is responsible for protein metabolism including growth through regulation of gene expression and protein metabolism [7].

There is a positive correlation between selenium levels in the body and level of circulating 3,3'-5 T3 [1]. Low selenium levels in the body have been observed to lead to increased T4 in circulation, but a reduction of 3,3'-5 T3 and Thyrotropin TSH. Other studies have shown the inverse relationship between the level of selenium in the body and level of thyroglobulin and serum concentration of 3,3'-5 T, and T4 [12]. While studies $[16,13]$ have shown inverse relationship between selenium levels in the human body and thyroid volume, and thyroid tissue damage, in women but not in men.

Besides DIOs, the family of Iso-forms of glutathione peroxides (cGPX, pGPX) and Thioredoxine reductases (TRrX) is associated with thyroid gland protection [7]. In presence of iodine, thyroxine production requires $\mathrm{H}_{2} \mathrm{O}_{2}$ [7] the production of which is generated by TSH up on stimulation by phospholipase $\mathrm{PIP}_{2}-\mathrm{IP}_{3}-\mathrm{Ca}^{2+}$ Cascade [21, 22], since the thyroxine biosynthesis by the follicles requires a high amount of $\mathrm{H}_{2} \mathrm{O}_{2}$ is produced, [10] the excess of which leads to accumulation of radicals and needs to be mopped out to avoid peroxidation of the thyrocytes [7]. This implies that sufficient intake of selenium is needed to ensure thyroid hormone production and thyroid tissue protection by cGSHpx and TxnrD. Some studies have reported association of multiple thyroid nodule risk, low volume of thyroglobulin in adult women with low serum selenium Administration of selenium has shown increase in weight in children who are HIV positive (as shown in Figure 1) below perhaps reflecting effect on thyroid gland.

The objective of this study was investigate the effect of selenium on weight change in HIV 1 positive pre-puberty, ARV naive children of different sexes (Tick-phenomenon).

\section{Materials and Methods}

\subsection{Sampling Criteria}

Fifty children were recruited to the study from Nyamasaria in Kisumu County. The children were enrolled into the study if they were between from age 3 and 16 years and the consent given by their guardians, and those who were HIV positive in WHO stage three and below. They were excluded if they were below three years and above 16 years didn't have guardian consent and were in HIV WHO stage four. Computer generated random numbers was used to select the required number after consultation with authorities at the center.

\subsection{Sample Size Determination}

The sample size was determined by Epi Info version 6 for cohort studies in which the required minimum sample size at $95 \%$ Confidence interval and precision/power of $80 \%$ and prevalence of HIV amongst the study subjects $15 \%$ (KAIS, 2007), Relative Risk of 5\% the number in test group is given 
as 13 while for the controls at 13 the minimum sample size being

\subsection{Ethical Consideration}

Approval to conduct the study was given by Kenyatta National Hospital Ethics Research and Standard committee. All patients who met the criteria were given equal chance to be enrolled in the study. Informed consent was given by the guardians/parents and the information collected was kept confidential.

\subsection{Administering of Selenium to the Children}

The participants on test were assigned to, and received $50 \mu \mathrm{g}$ selenium (yeast) for up to 6 months. The dose was about a half of tolerable upper limit for children which is $100 \mu \mathrm{g}$ (yeast selenium) per day hence considered safe. Every week an evaluation of the intervention was done and replenishment of the selenium stock and to monitor the compliance with the treatment. At 3 months intervals, the research assistants collected data on Weight to the nearest $0.1 \mathrm{~kg}$.

\subsection{Data Collection}

Interview of Guardians: At baseline an interview of Childrens' guardians were done by trained research assistants who collected data of children's' socioeconomic status and those of their guardians using structured questionnaire.

Measuring Children' Weight: The weight of the children were collected by electronic personal scale (model 1001) as was earlier suggested [15]. The scale was put on a flat surface and the reading adjusted to zero. Each child was told to stand on the scale evenly with barefoot wearing minimum clothing. The reading was taken in Kilograms.

\section{Results}

The mean age of the group on selenium was $7.7 \pm 3.4$ years, while for matched controls it was $8.8 \pm 3.2$ years. In matched controls $57.01 \%$ were girls while $42.09 \%$ were boys.

Table 1. Age and Sex distribution of study subjects.

\begin{tabular}{llll}
\hline & Selenium group & Control group & p-value \\
\hline Mean age group & $7.7 \pm 3.4$ & $8.8 \pm 3.2$ & 0.185 \\
Sex & & & \\
Girls & $22(42.9 \%)$ & $20(57.01 \%)$ & 0.853 \\
Boys & $12(35.3 \%)$ & $15(42.9 \%)$ & \\
Median weight & $18(10.9 \%)$ & $19(9.5 \%)$ & \\
\hline
\end{tabular}

Table 2. Change in WAZ Score among children on Test and Control.

\begin{tabular}{|c|c|c|}
\hline \multicolumn{3}{|c|}{ WAZ Scores For Test and Control } \\
\hline & Se WAZ & Control WAZ \\
\hline \multicolumn{3}{|c|}{ Three months } \\
\hline $3-5$ yrs & $0.15 \pm 1.110$ & $-2.01 \pm 0.160$ \\
\hline $6-8$ yrs & $0.006 \pm 1.22$ & $-1.63 \pm 0.051$ \\
\hline $9-16$ yrs & $0.19 \pm 0.880$ & $-2.95 \pm 3.010$ \\
\hline \multicolumn{3}{|c|}{ Six months } \\
\hline $3-5 y r s$ & $1.10 \pm 0.890$ & $-2.95 \pm 0.160$ \\
\hline $6-8 y r s$ & $-0.63 \pm 0.76$ & $-2.76 \pm 1.150$ \\
\hline $9-16 y r s$ & $0.97 \pm 1.22$ & $-2.30 \pm 1.240$ \\
\hline
\end{tabular}

There was a progressive increase in weight for age $\mathrm{Z}$ score among the children on test in all age groups, 3-5years $(1.20 \pm 2.45), 6-8$ years $(0.19 \pm 0.88)$ and age groups $9-16$ years $(-0.97 \pm 1.22)$ suggesting growth, while in the matched controls there was a decrease in weight for age $\mathrm{Z}$ score in different age groups 6-8 years $(-2.949 \pm 3.01)$, and 9-16 ($2.30 \pm-1.24)$ at six months compared to baseline suggesting wasting. Over all there was wasting among the children on control at six months in all age categories. Taking cu-off point of -2.00 SDs as a measure $100 \%$ of children on control group were wasted at six months. All the children on selenium non show wasting as all age groups at six months the WAZ score was above the cut-off point of -2SDs.

Further analysis of difference in weight for age $\mathrm{Z}$ score between test group and controls show there was a significant statistical difference $\{(\mathrm{df} 5,12)=5.758, \mathrm{p}=0.006\}$.

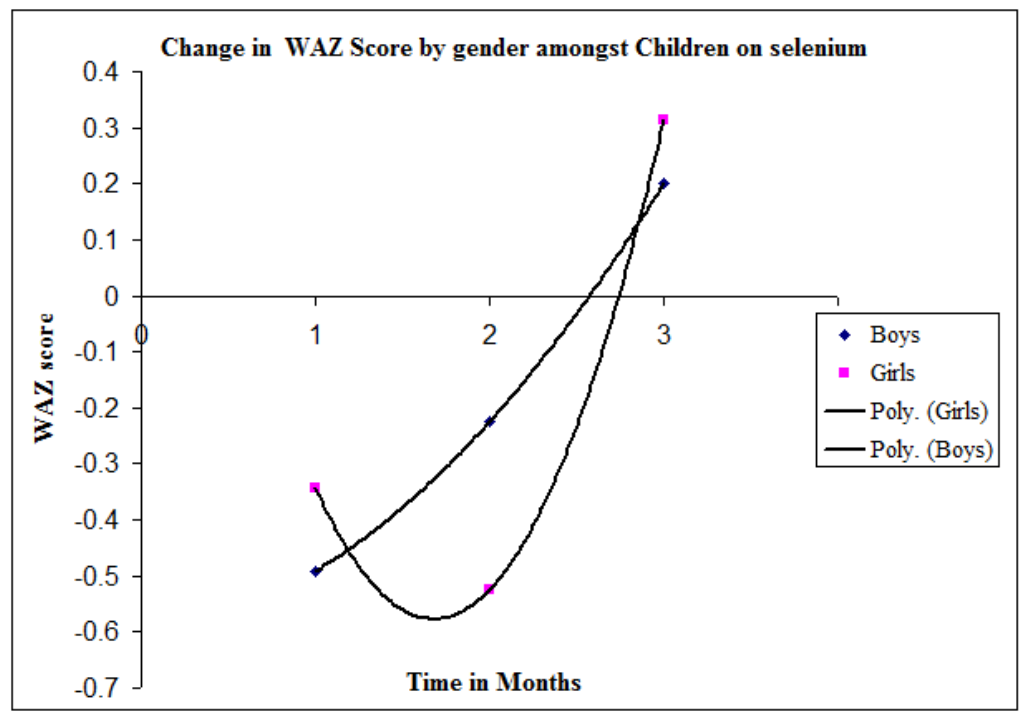

Figure 1. Effect of Selenium on WAZ of HIV positive Children (Tick phenomenon). 
Figure 1 Shows change in WAZ among the children on selenium. Weight for age Score among different sexes of the test group increased compared to the baseline with girls score having higher increase compared to boys (Tick Phenomenon) as shown in figure above WAZ for girls increased from 0.343 to 0.314 and boys from -0.491 to 0.201 . Among the controls the WAZ scores did not show much change a cross the gender categories The WAZ for girls changed from 2.536 to -0.440 and boys from -2.047 to -2.039 at six months.

\subsection{Change of WAZ by Sex and Age for Children on Control}

There was little or no change in weight for age $\mathrm{Z}$ score for children in control of boys in all age categories. The boys in age categories of 9-15 years had a decrease of WAZ from 1.37 to -2.08 for the 3-5 years old and 9-15 years old boys the WAZ remained below the cut-off point $(-2 . .00)$ at six months. For the girls on control there was a slight increase of WAZ score in all age categories 6-8 years from 1.87 to -1.32 and in age groups $9-15$ years from -2.50 to 0.001 at six months.

\subsection{Change of WAZ by Sex and Age for Children On Selenium}

The weight for age $\mathrm{Z}$ score for boys on selenium had a slight increase or remained the same at six months. The boys age group of 3-5 years had an increase from -1.93 to 2.09 at six months, 9-16byears had a slight decrease from -0.995 to 1.487. All age categories were above cut-off point of -2.00 at six months. Among the girls on selenium there was slight decrease among the age groups 3-5 years from 0.539 to 0.274 at six months. There was an increase from 0.119 to 0.312 in age group 6-8 years and from -1.678 to 0.904 in age groups 9-16 years. All categories were above WAZ score cutoff point at six months.

\section{Discussion}

WAZ for children in both groups were established at baseline. The cut-off of -2.00 standard deviation WHO was used to determine wasting among the children. The children with -2.00 SDs are normally considered wasted by WHO standards [15]. Over all $49 \%$ of children were established to be underweight at six months, with those in control group more wasted at $48 \%$ compared to test group at $1 \%$ at six months. Other studies [24] found that selenium forms active site of iodothyronine deiodinases which activate and deactivate thyroid hormones $T_{3}$ which is enhanced by sex hormone estradiol. These hormones are responsible for protein synthesis and deposition in the body. In this study there was significant increase in WAZ of children on selenium concurring with [24]. these findings therefore do confirm that intake of selenium did increase the body weight hence the WAZ in HIV positive children. this observation also confirmed by observation made by Niekerk et al in 2002 in south Africa.
HIV infection is accompanied by progressive nutrition alteration and pathological changes with critical pediatric HIV patients WAZ cut-off point being -2SDs [15]. This point marks beyond which there is increase in mortality among the patients. The observation in this study showed that all the children on control were below the cut-off point WAZ at six months hence were wasted and the condition if unchecked was likely to develop to fully blown AIDS.

\section{Conclusion}

From the study in can be concluded that intake of yeast selenium by treatment naïve HIV1 positive children led to significant increase in WAZ for both boys and girls (p. 0.05). Where are as in Boys the increase was gradual, in girls there was initial decrease followed by sudden steep increase (Tick Phenomenon) perhaps due to effects of hormone Estradiol.

\section{References}

[1] Arthur JR, Beckett GJ, (1988) Selenium deficiency and thyroid hormones metabolism. Selenium in biology and Medicine. New Yolk, NY; Springer-Verlag,; 90-5.

[2] Fairweather-Tait S. J, Yongping Bao, Martin R. Broadley, Rachel Collings, Dianne Ford, John E. Hesketh, and Rachel Hurst (2011) Selenium in Human health and disease: Antioxidant and redox Signaling 14: 7, 1337-1367.

[3] Burk R F, Hill K E (2011) Importance of Seleno-Protein P and Selenium Homoeostasis for maintaining Integrity And Function of The Brain and Other Tissues IN Banuelosn G, Lin ZQ Eds: SELENIU Global perspectives of Impacts on Humans, Animals and thev Environment: University Science of Science and Technology of China Press: 31.

[4] Otieno S. B. Were F N, Kabiru W, (2014) Study of Selenium content of foods in high HIV prevalent Community, A case Study in Pala Bondo District Kenya (in) Banuelos, Lin (Eds) Selenium in the Environment and Human Health, CRC Press, Taylor and Francis, London 64-65, ISBN 978-1-138-000179.

[5] Rayman M P, Alexander J Thompson, Bram Bekaert, Janet Catterick, Rachel Galassini, Emma Hall, Margaret WarrenPerry, and Geoffrey J Beckett (2008) Randomized controlled trial of the effect of selenium supplementation on thyroid function in the elderly in the United Kingdom1-American Journal of Clinical Nutrition: 87, 370-378.

[6] Ventura M, Miguel Melo, Francisco Carrilho (2016); Selenium and Thyroid Disease: From Pathophysiology to Treatment: Hindawi International Journal of Endocrinology: 2017: 1-9.

[7] Kohrle J (2005). Selenium and the control of Thyroid hormone metabolism. Thyroid, 15; 841-853.

[8] Contempre. B, de Escobar GM, Denef J F (2004) Thyocyanate induces cell necrosis and fibrosis in selenium experimental model for myxedematous endemic cretinism in central Africa, Endocrinology, 145; 994-1002. 
[9] Méplan, C.; Nicol, F., Burtle, B. T., Crosley, L. K.; Arthur, J. R.; Mathers, J. C.; Hesketh, J. E (2009): Relative Abundance of Selenoprotein P Isoforms in Human Plasma Depends on Genotype, Se Intake, and Cancer Status. Antioxidants. Redox Signal. 11, 2631-2640.

[10] Ekholm R, Bjorkman U (1997). Glutathione peroxidase degrades intracellular hydrogen peroxide and thereby inhibits intracellular protein iodination in thyroid epithelium. Endocrinology, 138; 2871-2878.

[11] Hussain MA, Schmitz O, Jorgensen JO, Christiansen JS, Weeke J, Schmid C, Froesch ER (1996) Insulin-like growth factor I alters peripheral thyroid hormone metabolism in humans: comparison with growth hormone. European Journal of Endocrinol 134: 563-567.

[12] Ravalgia G, Forti P, Maioli F, (2000). Blood micronutrient and thyroid hormone concentrations in the oldest-old. Journal of clinical endocrinology Metabolism; 85: 2260-5.

[13] Zagrodzki P, Szmigiel H, Ratajczac R (2000), The role of selenium in iodine metabolism in children with goiter. Environmental health Perspectives; 108: 67-71.

[14] Glatre E, Mracova A, Lener J, Vobecky M, Egertova E, Mysliveckova M (1995) Study of distribution and interraction of selenium and arsenic in rat thyroid. Biology of trace elements research; $49 ; 177-85$.

[15] Cogill U. M (1997) Cited by Harold Foster, The Distribution of Selenium and Mortality Owing to Acquired Immune Deficiency Syndrome in Continental United States Biological Trace Element Research, 56; 43.

[16] Derumeaux. H, Valeix. P, Castetbon. K, (2003) Association of selenium with thyroid volume and echostructure in 35 to 60 year old French adults. European Journal of Endocrinology, $148 ; 309-315$.
[17] Diplock. A. T. T., (1993) Indexes of Selenium Status in Human Populations. American Journal of Clinical Nutrition 57: 256-258.-61.

[18] Tani LSK, Nouria Dennouni-Medjati, Benoit Toubhans Laurent Charlet (2000);. Selenium Deficiency_From Soil to Thyroid Cancer; Applied Science; 10; 5368.

[19] Harper. H. A., Rodwel V. W., Mayes P. A. (1977), Review of Physiological Chemistry 16th Edition, 140-141.

[20] Clerk. M. L., Harvey. D. G., Humphreys. D. J., (1981) Veterinary Toxicology, Second Edition, English Language Book Society and Bellaire Tendal; 70-72.

[21] De Deken X, Wang D, Dumont JE, Miot F 2002 Characterization of ThOX proteins as components of the thyroid $\mathrm{H}_{2} \mathrm{O}_{2}$-generating system. Experimental Cell Research 273: 187-196.

[22] Caillou B, Dupuy C, Lacroix L, Nocera M, Talbot M, Ohayon R, Deme D, Bidart JM, Schlumberger M, Virion A 2001 Expression of reduced nicotinamide adenine dinucleotide phosphate oxidase (ThoX, LNOX, Duox) genes and proteins I $\mathrm{n}$ human thyroid tissues; Journal of Clinical Endocrinol Metabolism 86: 3351-3358.

[23] Berry, M. J. Insights into the hierarchy of selenium incorporation. National. Genetics. 2005, 37, 1162-1163.

[24] Gibson RS (2005); Principles of Nutritional Assessment Oxford; Oxford University Press UK.

[25] Whanger, PD, Ip, C, Polan, CE, Uden, PC \& Wilbaum, G (2000) Tumorigenesis, metabolism, speciation, bioavailability and tissue deposition of selenium in selenium-enriched ramps (Allium tricoccum). J Agric Food Chem 48, 5723-5730. 